\title{
IMPACT OF DRYING METHOD ON ANTIOXIDANT, ANTI-DIABETIC, AND ANTI-PROLIFERATION ACTIVITIES OF CIRSIUM SETIDENS IN VITRO
}

\author{
H.F. GuO and M.H. WANG* \\ Department of Medical Biotechnology, College of Biomedical Science, Kangwon National University, Chuncheon, \\ 24341. Republic of Korea
}

(Received: 7 March 2017; accepted: 30 May 2017)

\begin{abstract}
This study investigated the influences of drying method (oven-, freeze-, and shade-drying) and extraction solvent (ethanol and water) on the bioactivities of Cirsium setidens. Antioxidant activity was evaluated by DPPH radical scavenging ability, anti-diabetic activity was determined by the inhibitory activity of two enzymes: $\alpha$-glucosidase and $\alpha$-amylase, while anti-proliferation activity was assessed by MTT assay of three human cancer cell lines (KB, A549, and PC-3). Results indicated that bioactivities were extremely affected by solvent; water extracts contained more phenolics, exhibited strong anti-diabetic effect, but no activity of anti-proliferation, while the ethanolic extracts rich in flavonoids showed profound DPPH radical scavenging and anti-proliferation ability, yet low activity of antidiabetes. Among the drying methods, freeze-drying extracts preserved more flavonoids and exhibited better activity of anti-proliferation, while shade-drying extracts contained higher phenolics and showed stronger activity on antidiabetes, oven-drying gave the lowest content of phenolics. Hence, antioxidant and anti-diabetic effects were positively related to phenolic content, meanwhile an extremely significant correlation coefficient had been found between anti-proliferation activity and flavonoid content, it can be concluded that drying method and extraction solvent affect bioactivities by phenolic and flavonoid contents.
\end{abstract}

Keywords: antioxidant, anti-diabetic, anti-proliferation, Cirsium setidens, drying method

Cirsium setidens (Asteraceae family), is one of the thistles native in Gangwon do, Republic of Korea. Cirsium setidens is a nutrient rich plant abundance of calcium and vitamins, therefore residents are accustomed to eating leaves and stems of the plant as vegetables. It is also employed as a folk medicine to treat hematemesis, hypertension, and hematuria.

Since $C$. setidens exhibits plenty of benefits, it is worthy to explore the use of $C$. setidens in cosmetics, medical and functional food. Generally, plants are marketed in forms of extracts or powders produced from dried plants. The removal of water can be classified by temperature into three categories: drying at high, normal, or low temperature. The heating process evaporates moisture on the surface and forces the inside moisture to travel to the surface, there it is evaporated and freeze drying sublimates the moisture directly into vapour. Heating accelerated the drying process and prevented reproduction of microorganisms, while at normal temperature the process needs more time and freezing has a higher cost (RAGHAVAN \& ORSAT, 2007). However, drying process seriously affected the bioactivities of the product through enzymatic degradation, volatilization, and decomposition (MA et al., 2013). Therefore, a process optimization should be carried out to provide the best nutritional values and functional activity in the final products. According to a study, hot temperature treatment provides more bioactive compounds than freeze-drying treatment in case of olive leaves

* To whom correspondence should be addressed.

Phone: +82-33-250-6486; fax: +82-33-259-5644; e-mail: mhwang@kangwon.ac.kr

0139-3006 (C) 2018 Akadémiai Kiadó, Budapest 
(Ahmad-QAsem et al., 2013). Whereas, ChAn and co-workers (2009) reported that freezedrying provided a significant increase in total phenolics content, ascorbic acid equivalent antioxidant activity, and reducing power activity opposite to other methods. In this case, whether the drying process of $C$. setidens affected bioactivity is worthy to be investigated. To our knowledge, there is no literature focusing on this field, and we intend to use oven-, freeze-, and shade-drying methods to dehydrate fresh C. setidens leaves and to compare the bioactivities of their ethanol and water extracts.

\section{Materials and methods}

\subsection{Chemicals and reagents}

Folin-Ciocalteu reagent, aluminium chloride hexahydrate, tannic acid, quercetin, acarbose, 3-(4,5-dimethylthiazol-2-yl)-2,5-diphenyltetrazolium bromide (MTT), 1,1-diphenyl-2picrylhydrazyl (DPPH), $p$-nitrophenyl- $\alpha$-d-glucopyranoside (pNPG), dinitrosalicylic acid (DNS), $\alpha$-glucosidase from Saccharomyces cerevisiae, and $\alpha$-amylase from porcine pancreas were purchased from Sigma (St. Louis, MO, USA). RPMI 1640, DMEM (high glucose), fetal bovine serum (FBS), and trypsin-EDTA were acquired from Hyclone (Thermo Scientific, Waltham, MA, USA). The culture supplies were purchased from SPL Brand Products (SPL, Republic of Korea). All chemicals or reagents were of analytical grade.

\subsection{Materials}

Fresh C. setidens leaves were obtained in Gangwon do, Republic of Korea. The fresh plants were divided into three parts and dried by oven-drying, freeze-drying, and shade-drying. Oven-drying was carried out in an electric thermo static drying oven at $55^{\circ} \mathrm{C}$. Freeze-drying was done in a freeze dryer (FDE-0350, Humanlab instrument, Republic of Korea) at $-48^{\circ} \mathrm{C}$, and the shade-drying was in a draughty house avoid of sunlight at room temperature $\left(25 \pm 2{ }^{\circ} \mathrm{C}\right)$. The dried plants were ground in a rotary mill and extracted by ethanol or water.

\subsection{Total phenolic and flavonoid content}

Total phenolic content was evaluated following the method reported by SINGH and co-workers (2002) with modification. Sample solution reacted with $10 \%$ Folin-Ciocalteu reagent and $7.5 \%$ sodium carbonate for $30 \mathrm{~min}$ in dark, the absorbance was detected at $750 \mathrm{~nm}$. Tannic acid was chosen as the standard and total phenolics content was expressed as mg tannic acid equivalent/g (mg TAE/ g).

Total flavonoid content was detected by the mixture of aluminium chloride solution and sample ethanol solution. The absorbance of the solution was measured at $405 \mathrm{~nm}$ after $1 \mathrm{~h}$. Quercetin was employed as the standard, thus total flavonoids content was expressed as mg quercetin equivalent/g (mg QE/g).

\subsection{DPPH radical scavenging activity}

DPPH scavenging activity was measured according to the method of ERKAN and co-workers (2008) with a slight modification. Five hundred microlitres of $0.2 \mathrm{mM}$ DPPH solution (in methanol) was mixed with the same volume of sample solution (in methanol) at various 
concentrations. It was left to stand for $30 \mathrm{~min}$ in darkness at room temperature after vortexing, then the absorbance was measured at $515 \mathrm{~nm}$ using a microplate spectrophotometer reader (ELx800TM, BioTek, Winooski, VT, USA). $\alpha$-Tocopherol was used as positive control.

\subsection{Alpha-glucosidase inhibitory assay}

Alpha-glucosidase activity was measured by a modified method described by КІм and соworkers (2005), $4 \mathrm{mM} \mathrm{pNPG} \mathrm{substrate} \mathrm{solution} \mathrm{(pH} \mathrm{6.9)} \mathrm{was} \mathrm{added} \mathrm{to} \mathrm{the} \mathrm{mixture} \mathrm{of} \mathrm{extract}$ and $\alpha$-glucosidase solution after pre-incubation. The reaction was stopped after $20 \mathrm{~min}$ incubation at $37^{\circ} \mathrm{C}$ by $0.1 \mathrm{M} \mathrm{Na}_{2} \mathrm{CO}_{3}$ and measured at $400 \mathrm{~nm}$. Acarbose was used as positive control and the blank was distilled water.

\subsection{Alpha-amylase inhibition assay}

Alpha-amylase inhibition assay was carried out according to McCue and ShetTy (2004). After the pre-incubation of the mixture of extract and $\alpha$-amylase, $2 \%$ starch was added, and the mixture was incubated for $5 \mathrm{~min}$. DNS (pH 6.8) was added and the reaction was stopped by heating at $100{ }^{\circ} \mathrm{C}$ for $15 \mathrm{~min}$. The absorption was measured at $540 \mathrm{~nm}$ (room temperature). Acarbose was employed as positive control and the blank was distilled water.

\subsection{Anti-proliferation activity of the cancer cells}

KB (human oral carcinoma cell), A549 (human lung cancer cell), and PC-3 (human prostate cancer cell) cell lines were purchased from Korean Cell Line Bank (KCLB, Seoul, Republic of Korea) and cultured as the supplier described. The anti-proliferation activity was determined by MTT assay with 24,48 , or $72 \mathrm{~h}$ treatment of indicated concentration of extracts, followed by the procedure reported by VAN MEerLoo and co-workers (2011) and calculated by the percentage of untreated control.

\subsection{Statistical analysis}

The results were expressed in terms of mean $(\mathrm{n}=3) \pm$ standard deviation. Statistical analysis was conducted by SPSS 21 (SPSS Institute, Cary, NC, USA) through one-way analysis of variance (ANOVA followed by Duncan's multiple-range test) to determine the significant differences between the groups.

\section{Results and discussion}

\subsection{Antioxidant activity}

DPPH is a free radical commonly employed to measure the antioxidant activity. As shown in Figure 1, shade-drying ethanol extract (SDE) and oven-drying ethanol extract (ODE) almost abolished the free radical completely at $200 \mu \mathrm{g} \mathrm{ml}^{-1}$, followed by freeze-drying ethanol extract (FDE), oven-drying water extract (ODW), freeze-drying water extract (FDW), and shade-drying water extract (SDW). Among the three drying methods, oven-drying displayed greater activity, shade-drying results varied with extraction solvent, and freeze-drying exhibited less activity in scavenging DPPH free radical. 




Fig.1. DPPH free radical scavenging activity of $C$. setidens extracts

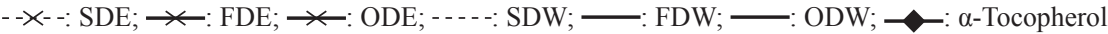

Table 1. Alpha-glucosidase and $\alpha$-amylase inhibitory activity of $C$. setidens extracts

\begin{tabular}{|c|c|c|c|c|c|c|}
\hline \multirow[t]{2}{*}{ Extracts } & \multicolumn{3}{|c|}{ Alpha-glucosidase inhibitory activity (\%) } & \multicolumn{3}{|c|}{ Alpha-amylase inhibitory activity (\%) } \\
\hline & $0.1 \mathrm{mg} \mathrm{ml}^{-1}$ & $0.2 \mathrm{mg} \mathrm{ml}^{-1}$ & $0.4 \mathrm{mg} \mathrm{ml}^{-1}$ & $0.1 \mathrm{mg} \mathrm{ml}^{-1}$ & $0.2 \mathrm{mg} \mathrm{ml}^{-1}$ & $0.4 \mathrm{mg} \mathrm{ml}^{-1}$ \\
\hline FDE & - & $0.86 \pm 0.24 \mathrm{e}$ & $1.37 \pm 0.31 \mathrm{f}$ & $2.65 \pm 0.76 f$ & $3.82 \pm 1.34 \mathrm{f}$ & $5.65 \pm 0.76 f$ \\
\hline SDE & - & $1.12 \pm 0.3 \mathrm{e}$ & $2.19 \pm 0.08 \mathrm{e}$ & $5.65 \pm 1.11 \mathrm{e}$ & $7.55 \pm 1.02 \mathrm{e}$ & $10.65 \pm 1.11 \mathrm{e}$ \\
\hline ODE & - & - & - & $1.98 \pm 0.24 \mathrm{f}$ & $2.16 \pm 0.98 f$ & $3.98 \pm 0.24 \mathrm{~g}$ \\
\hline FDW & $58.14 \pm 2.28 \mathrm{c}$ & $64.63 \pm 1.01 \mathrm{c}$ & $72.22 \pm 1.32 \mathrm{c}$ & $54.76 \pm 1.13 \mathrm{c}$ & $63.21 \pm 0.79 \mathrm{c}$ & $75.20 \pm 1.10 \mathrm{c}$ \\
\hline SDW & $68.44 \pm 1.67 \mathrm{~b}$ & $74.45 \pm 1.35 b$ & $80.87 \pm 2.78 b$ & $62.33 \pm 0.58 b$ & $78.48 \pm 0.65 b$ & $81.49 \pm 1.41 \mathrm{~b}$ \\
\hline ODW & $49.38 \pm 0.92 \mathrm{~d}$ & $55.34 \pm 2.11 d$ & $62.87 \pm 2.54 \mathrm{~d}$ & $43.98 \pm 0.19 \mathrm{~d}$ & $50.77 \pm 0.81 \mathrm{~d}$ & $59.32 \pm 1.54 \mathrm{~d}$ \\
\hline Acarbose & $78.09 \pm 1.02 \mathrm{a}$ & $82.76 \pm 1.98 \mathrm{a}$ & $88.97 \pm 1.61 \mathrm{a}$ & $74.54 \pm 0.74 \mathrm{a}$ & $85.98 \pm 1.93 \mathrm{a}$ & $91.21 \pm 2.04 \mathrm{a}$ \\
\hline
\end{tabular}

-: No activity; a,b,c,d,e,f: Means sharing the same letter indicate no difference with $\mathrm{P}<0.05$

\subsection{Anti-diabetic activity}

The anti-diabetic effect is determined in vitro by $\alpha$-glucosidase inhibition and $\alpha$-amylase inhibitory activity. SDW showed the strongest inhibitory activity in $\alpha$-glucosidase and $\alpha$-amylase inhibition assay of $80.87 \%$ and $81.49 \%$, respectively, maximum inhibitory activity at $0.4 \mathrm{mg} \mathrm{ml}^{-1}$, and the ODE showed the lowest inhibitory activity with no inhibition effect of $\alpha$-glucosidase and only $3.98 \%$ of $\alpha$-amylase inhibition at $0.4 \mathrm{mg} \mathrm{ml}^{-1}$. According to the data obtained, ethanol extracts produced by any drying method exhibited little inhibitory 
activity, and simultaneously, water extracts showed a remarkable inhibitory activity in both assays. Furthermore, comparison among the drying methods revealed that shade-drying possessed stronger inhibitory activity, independently of the extraction solvent, followed by freeze- and oven-drying.

\subsection{Anti-cancer activity}

Anti-cancer activity was measured by the anti-proliferative effect of three cancer cell lines with MTT colorimetric assay. It can be seen in Figure 2 that FDE inhibited the proliferation of KB cancer cells effectively, followed by SDE and ODE, but water extracts (SDW, FDW, and ODW) showed scarcely any inhibitory activity. The same trends were detected in A549 and PC-3 cancer cell lines, and the extracts inhibited cellular proliferation in a concentration and time dependent manner.
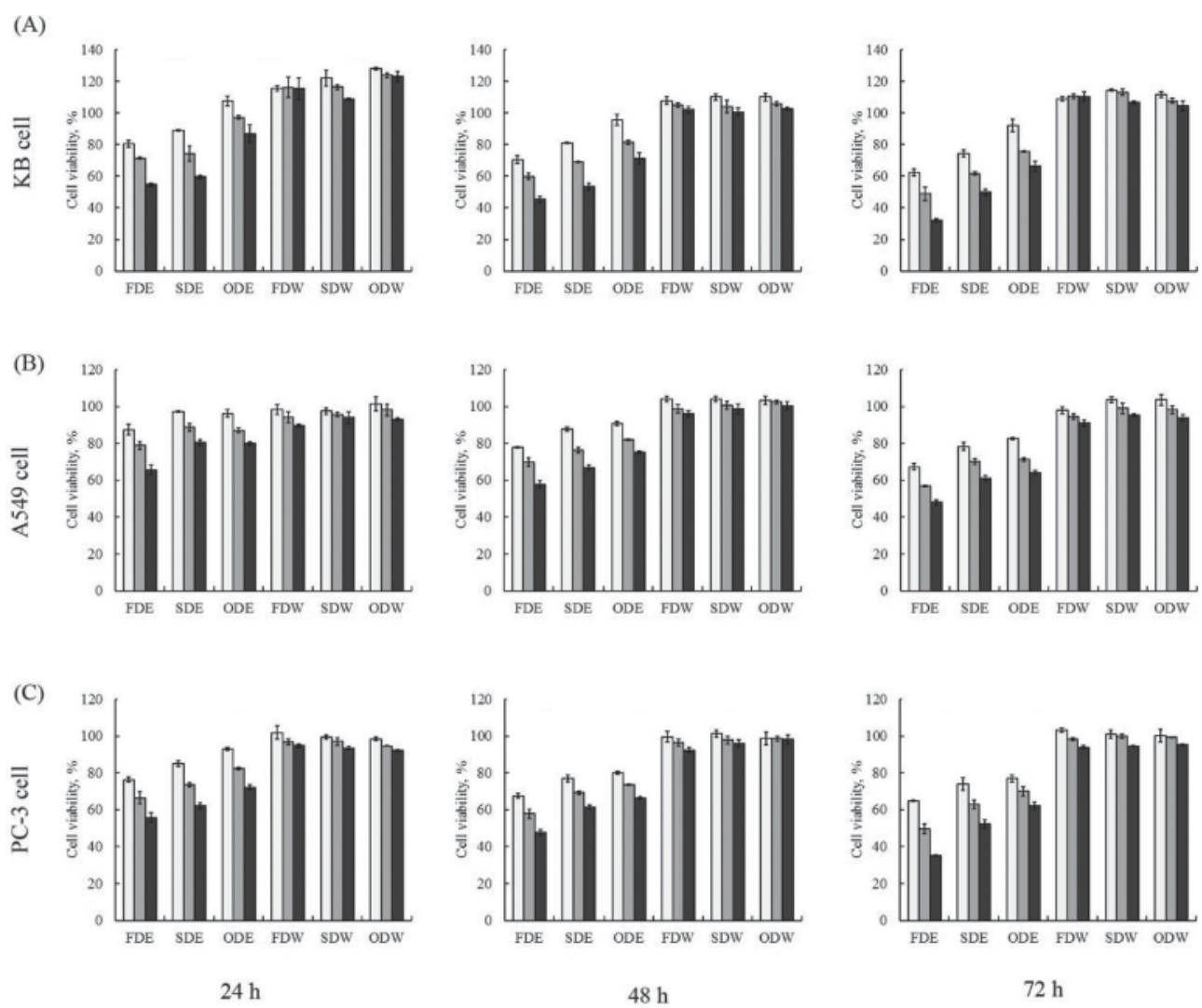

Fig. 2. Anti-proliferative effect of $C$. setidens on cancer cells after 24,48 , and $72 \mathrm{~h}$. KB human oral cancer cells (A), A549 human lung cancer cells (B), and PC-3 human prostate cancer cells (C) were exposed to 200, 400, and $800 \mu \mathrm{g} \mathrm{ml}^{-1}$ of $C$. setidens extracts for 24,48 , and $72 \mathrm{~h}$ $\square: 200 \mu \mathrm{g} \mathrm{ml}^{-1} ; \square: 400 \mu \mathrm{g} \mathrm{ml}^{-1} ; \mathbf{\square}: 800 \mu \mathrm{g} \mathrm{ml}^{-1}$ 
Table 2. Total phenolic and flavonoid content of $C$. setidens extracts

\begin{tabular}{lcc}
\hline Extracts & Phenolics $(\mathrm{mg}$ TAE $/ \mathrm{g})$ & Flavonoids $(\mathrm{mg}$ QE/g) \\
\hline FDE & $28.76 \pm 1.21 \mathrm{bc}$ & $15.36 \pm 0.42 \mathrm{a}$ \\
SDE & $29.58 \pm 0.84 \mathrm{~b}$ & $13.21 \pm 0.13 \mathrm{~b}$ \\
ODE & $24.29 \pm 0.41 \mathrm{~d}$ & $11.58 \pm 0.24 \mathrm{c}$ \\
FDW & $31.15 \pm 0.37 \mathrm{~b}$ & $9.43 \pm 0.21 \mathrm{~d}$ \\
SDW & $32.16 \pm 0.62 \mathrm{a}$ & $8.93 \pm 0.36 \mathrm{~d}$ \\
ODW & $28.83 \pm 0.18 \mathrm{c}$ & $7.81 \pm 0.65 \mathrm{e}$ \\
\hline
\end{tabular}

a,b,c,d,e : Means sharing the same letter indicate no difference with $\mathrm{P}<0.05$

\subsection{Total phenolic and flavonoid contents}

FDE had the highest flavonoid content of $28.36 \pm 0.42 \mathrm{mg}$ QE/g, and SDW contained the highest amount of phenolics of $36.16 \pm 0.62 \mathrm{mg} \mathrm{TAE} / \mathrm{g}$ (Table 1). Among ethanol extracts, FDE was abundant in flavonoids and SDE in phenolics, and the same results were found for water extracts. To compare between drying methods, freeze-dried extracts contained the largest volume of flavonoids, followed by shade- and oven-drying. Moreover, shade-dried extracts had higher phenolic contents, followed by freeze- and shade-drying.

Research on functional foods and ingredients has attracted attention in the recent years, supported by the increasing knowledge on natural products and pursuit of health by consumers. Plants are the main sources of the natural products or functional foods, the extracts of plants were demonstrated to be beneficial to human health, such as grape seed extract, which contains proanthocyanidin complexes and other antioxidants linked to a wide range of therapeutic properties (TORRES et al., 2002). Cirsium genus, which is often known as thistles, is used in traditional medicine for centuries to protect liver and stanch bleeding. The C. setidens had also been demonstrated by researchers to exhibit healthcare effects attributed to many compounds, like polyphenols and monosaccharides (LEE et al., 2006).

The bioactivity of the extracts from the plant is significantly influenced by the processing conditions, which already had been demonstrated in many reports (KiDMOSE et al., 2004). Different treatments affect differently the release of volatile compounds or the retention of bioactive compounds. Oven-drying is widely used in the process, since it is low cost, and high temperature does inactivate various micro-organisms that helps maintaining sensorial quality. Higher antioxidant activity was observed in roasted coffee and oven-dried pumpkin flour compared to freeze-dried or shade-dried products, suggesting that heating generates Maillard-derived antioxidants and enhances the capacity of some compounds (QuE et al., 2008). However, it has also been reported that heating process impairs bioactive property and reduces the content of bioactive compounds (PATRAS et al., 2009). Although freeze-drying is considered to decrease the amounts of certain volatile components substantially like ovendrying, it keeps the medicinal properties of the fresh plant in many cases (DíAz-MАroto et al., 2002). Shade-drying normally preserves volatiles better than oven- or freeze-drying, but the still active enzymes of the fresh plant may cause degradation of bioactive compounds during the drying process (Hossain et al., 2010). These studies show that drying methods affect bioactivity in a variety of ways for different plants. As drying temperature affects composition profile of plant products, the proper temperature should be applied for each specific purpose. 
Table 3. The correlation coefficient between bioactivities and phenolic or flavonoid contents

\begin{tabular}{lcc}
\hline Extracts & Phenolics & Flavonoids \\
\hline DPPH scavenging activity & -0.556 \\
Alpha-glucosidase inhibitory activity & 0.742 & $-0.858^{*}$ \\
Alpha-amylase inhibitory activity & 0.698 & $-0.836^{*}$ \\
Anti-proliferation activity & 0.733 & $0.973^{* *}$ \\
\hline
\end{tabular}

$*: \mathrm{P}<0.05 ; * *: \mathrm{P}<0.01$

\# The correlation coefficient was calculated between $\mathrm{IC}_{50}$ value of DPPH scavenging activity and phenolics or flavonoids, the inhibitory activity of $800 \mu \mathrm{g} \mathrm{ml} \mathrm{m}^{-1}$ extracts on $\alpha$-glucosidase/ $\alpha$-amylase/KB cells and phenolics or flavonoids

In our study, anti-cancer activity showed an extremely significant correlation coefficient with flavonoid content $(\mathrm{R}=0.973)$, anti-diabetic activity was positively related to the phenolic content but was significantly negatively related to the flavonoid content, and antioxidant activity exhibited a positive correlation with phenolics (Table 3). The C. setidens was abundant in pectolinarin, pectolinarigenin, linarin, apigenin, diosmetin, which had been reported responsible for anti-cancer activity (Yoo et al., 2008). Based on our results, FDE exhibited the strongest inhibitory effect on cancer cell, probably due to the fact that freezedrying process reserves the highest amount of flavonoids, which was coincident with results of Pérez-Gregorio and co-workers (2011). Phenolics were used as inhibitors to decrease blood glucose by inhibiting the $\alpha$-glucosidase and $\alpha$-amylase activities, resulting the decline of carbohydrate hydrolysis (PANTIDos et al., 2014). SDW was found richer in phenolics, and therefore it exhibited better anti-diabetic activity. The DPPH free radical scavenging activity was reported to be related to both phenolic and flavonoid contents, but in our research, a negative correlation coefficient was found between DPPH scavenging activity and flavonoids, suggesting that in $C$. setidens leaves phenolics were the leading compounds responsible for antioxidant activity. LEE and co-workers (2002) reported that one of the major phenolics in C. setidens was $\alpha$-tocopherol, which also explains why ethanol extracts exhibited better antioxidant activity.

\section{Conclusions}

In conclusion, this study verified that $C$. setidens leaves had effective anti-diabetic and anticancer activities, but the drying methods and extraction solvents seriously affected bioactivity. According to our data, best extract was obtained by water solvent and shade-drying for antidiabetes and ethanol solvent and freeze-drying for anti-cancer, as solvent and drying method affected phenolic or flavonoid content. Additional research should focus on identifying responsible compounds of anti-diabetes or anti-proliferation properties.

\section{References}

Ahmad-Qasem, M.H., Barrajón-Catalán, E., Micol, V., Mulet, A. \& García-Pérez, J.V. (2013): Influence of freezing and dehydration of olive leaves (var. Serrana) on extract composition and antioxidant potential. Food Res. Int., 50, 189-196. 
Chan, E.W.C., Lim, Y.Y., Wong, S.K., Lim, K.K., Tan, S.P., Lianto, F.S. \& Yong, M.Y. (2009): Effects of different drying methods on the antioxidant properties of leaves and tea of ginger species. Food Chem., 113, 166-172.

Díaz-Maroto, M., Pérez-Coello, M. \& Cabezudo, M. (2002): Effect of different drying methods on the volatile components of parsley (Petroselinum crispum L.). Eur. Food Res. Technol., 215, 227-230.

Erkan, N., Ayranci, G. \& Ayranci, E. (2008): Antioxidant activities of rosemary (Rosmarinus officinalis L.) extract, blackseed (Nigella sativa L.) essential oil, carnosic acid, rosmarinic acid and sesamol. Food Chem., 110, 7682.

Hossain, M.B., Barry-Ryan, C., Martin-Diana, A.B. \& Brunton, N.P. (2010): Effect of drying method on the antioxidant capacity of six Lamiaceae herbs. Food Chem., 123, 85-91.

Kidmose, U., Hansen, S.L., Christensen, L.P., Edelenbos, M., Larsen, E. \& NørbÆK, R. (2004): Effects of genotype, root size, storage, and processing on bioactive compounds in organically grown carrots (Daucus carota L.). $J$. Food Sci., 69, S388-S394.

Kim, Y.M., Jeong, Y.K., WAng, M.H., Lee, W.Y. \& RheE, H.-I. (2005): Inhibitory effect of pine extract on $\alpha$-glucosidase activity and postprandial hyperglycemia. Nutrition, 21, 756-761.

Lee, S.H., Jin, Y.S., Heo, S.I., Shim, T.H., SA, J.H., Choi, D.S. \& Wang, M.H. (2006): Composition analysis and antioxidative activity from different organs of Cirsium setidens Nakai. Korean J. Food Sci. Technol., 38, $571-576$

Lee, W.B., Kwon, H.C., Cho, O.R., Lee, K.C., Chol, S.U., Baek, N.I. \& Lee, K.R. (2002): Phytochemical constituens of Cirsium setidens Nakai and their cytotoxicity against human cancer cell lines. Arch. Pharm. Res., 25, $628-635$.

MA, L., CHen, H., ZHU, W. \& WANG, Z. (2013): Effect of different drying methods on physicochemical properties and antioxidant activities of polysaccharides extracted from mushroom Inonotus obliquus. Food Res. Int., 50, 633-640.

McCue, P.P. \& Shetty, K. (2004): Inhibitory effects of rosmarinic acid extracts on porcine pancreatic amylase in vitro. Asia Pac. J. Clin. Nutr., 13, 101-106.

Pantidos, N., Boath, A., Lund, V., Conner, S. \& McDougall, G.J. (2014): Phenolic-rich extracts from the edible seaweed, Ascophyllum nodosum, inhibit $\alpha$-amylase and $\alpha$-glucosidase: Potential anti-hyperglycemic effects. $J$. Funct. Foods, 10, 201-209.

Patras, A., Brunton, N., Da Pieve, S., Butler, F. \& Downey, G. (2009): Effect of thermal and high pressure processing on antioxidant activity and instrumental colour of tomato and carrot purées. Innov. Food Sci. Emerg., 10, 16-22.

Pérez-Gregorio, M.R., Regueiro, J., González-Barreiro, C., Rial-Otero, R. \& Simal-Gándara, J. (2011): Changes in antioxidant flavonoids during freeze-drying of red onions and subsequent storage. Food Control., 22, 11081113.

QuE, F., MAO, L., FAng, X. \& Wu, T. (2008): Comparison of hot air-drying and freeze-drying on the physicochemical properties and antioxidant activities of pumpkin (Cucurbita moschata Duch.) flours. Int. J. Food Sci. Tech., 43, 1195-1201.

Raghavan, G. \& ORsat, V. (2007): Recent advances in drying of biomaterials for superior quality bioproducts. AsiaPac. J. Chem. Eng., 2, 20-29.

Singh, R.P., Chidambara Murthy, K.N. \& Jayaprakasha, G.K. (2002): Studies on the antioxidant activity of pomegranate (Punica granatum) peel and seed extracts using in vitro models. J. Agr. Food Chem., 50, 81-86.

Torres, J.L., Varela, B., García, M.T., Carilla, J., Matito, C., Centelles, J.J., Cascante, M., Sort, X. \& Bobet, R. (2002): Valorization of grape (Vitis vinifera) byproducts. Antioxidant and biological properties of polyphenolic fractions differing in procyanidin composition and flavonol content. J. Agr. Food Chem., 50, 7548-7555.

Van Meerloo, J., Kaspers, G.J. \& Cloos, J. (2011): Cell sensitivity assays: the MTT assay. -in: Cree, I.A. (Ed.) Cancer cell culture: Methods and protocols. Methods Mol. Biol., 731, 237-245.

Yoo, Y.M., NAM, J.H., Kim, M.Y., CHOI, J. \& PARK, H.J. (2008): Pectolinarin and pectolinarigenin of Cirsium setidens prevent the hepatic injury in rats caused by D-galactosamine via an antioxidant mechanism. Biol. Pharm. Bull., 31, 760-764. 